\title{
Is brain circuitry linked with early symptoms of autism spectrum disorder?
}

\author{
By Dr. Jessica Edwards
}

Researchers in San Diego, USA, have studied the relationship between brain network connectivity and emerging autism spectrum disorder (ASD) symptoms in toddlers aged $17-45$ months with $(n=24)$ or without $(n=23)$ ASD. Bosi Chen and colleagues collected functional MRI data from children during their sleep, and investigated intrinsic functional connectivity both within and between the resting-state functional networks. They found greater between-network functional connectivity involving visual and sensorimotor circuits in those with ASD compared to typically developing toddlers. This increase in visual-sensorimotor connectivity was associated with more autism symptoms.

"Our finding of greater connectivity between visual and sensorimotor networks may be related to the sensory processing abnormalities experienced by a large proportion of young children with ASD", explains Chen. "Early difficulties with sensory processing can also have a downstream effect on later problems with language and social skills."

This study is limited by its small sample size and the lack of appropriate measures of sensory processing abnormalities in ASD. The researchers also did not study whether there were any differences in sleep stage between the two groups when collecting the fMRI data. Nevertheless, this study constitutes the first characterization of large-scale restingstate functional networks in children of this age group with ASD.

"Our identification of differences in network connectivity in toddlers with ASD highlights the impact of dysfunctional connectivity within primary sensory circuits on autism symptomatology", says Chen. "These findings might potentially guide the development of interventions targeting these brain networks early in life, such as by using non-invasive neurostimulation techniques."

\section{Referring to:}

Chen, B., Linke, A., Olson, L., Ibarra, C., Reynolds, S., Müller, $R$-A., Kinnear, M. \& Fishman, I. (2020), Greater functional connectivity between sensory networks is related to symptom severity in toddlers with autism spectrum disorder. J. Child Psychol. Psychiatr. doi: 10.1111/ jcpp.13268.

\section{Glossary:}

Functional MRI: This technique measures metabolic activity in the brain by detecting changes in blood flow. This approach is possible because when an area of the brain is in use, the blood flow to that region increases.

Functional connectivity: The amount by which brain areas are thought to be connected, based on the fact they are active at the same time. 